\title{
Identification of Piperlongumine (PL) as a new E3 ligase ligand to induce targeted protein degradation
}

Jing Pei,,${ }^{\mathrm{a}, \dagger}$ Yufeng Xiao, ${ }^{\mathrm{b}, \dagger}$ Xingui Liu,,${ }^{\mathrm{a}} \dagger$ Wanyi Hu, ${ }^{\mathrm{b}}$ Amin Sobh, ${ }^{\mathrm{c}}$ Yaxia Yuan, ${ }^{\mathrm{a}}$ Shuo Zhou, ${ }^{\mathrm{a}}$ Nan Hua, ${ }^{\mathrm{a}}$ Samuel G Mackintosh, ${ }^{\mathrm{d}}$ Xuan Zhang, ${ }^{\mathrm{b}}$ Kari B. Basso, ${ }^{\mathrm{e}}$ Manasi Kamat, ${ }^{\mathrm{e}}$ Qingping Yang, ${ }^{\mathrm{a}}$ Jonathan D. Licht, ${ }^{\mathrm{c}}$ Guangrong Zheng, ${ }^{* \mathrm{~b}}$ Daohong Zhou, ${ }^{* \mathrm{a}}$ and Dongwen $\mathrm{Lv}^{*} \mathrm{a}$

${ }^{\text {a }}$ Department of Pharmacodynamics, College of Pharmacy, University of Florida, 1333 Center Drive, Gainesville, FL, 32610, United States.

${ }^{\mathrm{b}}$ Department of Medicinal Chemistry, College of Pharmacy, University of Florida, 1333 Center Drive, Gainesville, FL, 32610, United States.

${ }^{\mathrm{c}}$ Division of Hematology/Oncology, University of Florida Health Cancer Center, 2033 Mowry Road, Suite 145, Gainesville, FL 32610, United States.

${ }^{\mathrm{d}}$ Department of Biochemistry and Molecular Biology, College of Medicine, University of Arkansas for Medical Sciences, 4301 W. Markham Street, Slot 803, Little Rock, AR 72205, United States.

${ }^{\text {e }}$ Department of Chemistry, University of Florida, PO Box 117200, Gainesville, FL 32611, United States.

${ }^{\dagger}$ These authors contributed equally to this work.

* These authors jointly directed this work: Guangrong Zheng, Daohong Zhou, and Dongwen Lv

Abstract: PROteolysis Targeting Chimeras (PROTACs) are bifunctional molecules that degrade target proteins through recruiting E3 ligases. However, their application is limited in part because few E3 ligases can be recruited by known E3 ligase ligands. Through competitive activity-based protein profiling, we found that piperlongumine (PL), a natural product, binds multiple E3 ligases. To evaluate whether PL can be used as an E3 ligase ligand, we generated a series of PL and SNS-032 (a selective CDK9 
inhibitor) conjugates and found that the lead conjugate 955 can potently degrade CDK9 in a ubiquitin-proteasome dependent manner. In addition, 955 is more potent than SNS032 against various tumor cells in vitro. Through TurboID-based proteomics and mechanistic studies, we identified KEAP1 as the E3 ligase recruited by PL to degrade CDK9. These findings demonstrate that PL is a novel E3 ligase ligand that can be used to generate potent anticancer PROTACs.

Keywords: Piperlongumine, E3 ligase, PROTAC, KEAP1, CDK9

\section{Introduction}

PROteolysis TArgeting Chimeras (PROTACs) are potentially more potent anticancer therapeutics than small molecule inhibitors (SMIs) because they can degrade oncoproteins in an event-driven manner[1]. Moreover, compared to SMIs that only block the catalytic function of proteins of interest (POIs), PROTACs can further remove the scaffold function of POIs through inducing their degradation. Furthermore, PROTACs have the ability to target some previously considered undruggable proteins, such as transcription factors. For example, a potent signal transducer and activator of transcription 3 (STAT3) PROTAC has been generated and shown efficacy in vivo[2]. In addition, PROTAC-induced POI degradation is driven by the ternary complex formation and can be affected by the availability of lysine on a POI[3]. Therefore, PROTACs can be more specific/selective than their SMI predecessors. Because of these advantages, more than 10 PROTACs have been advanced to phase I or phase II clinical trials by the end of 2021[4]. The targets include androgen receptor (AR), estrogen receptor (ER), B-cell lymphoma extra large (BCL-xL), bruton tyrosine kinase (BTK), bromodomain-containing protein 9 (BRD9), interleukin-1 receptor-associated kinase 4 (IRAK4), STAT3, and tropomyosin receptor kinase (TRK)[4].

Despite the great progress in the field, there are still some obstacles that prevent PROTACs from being more useful[5]. Among them, to date only a few E3 ligases and ligands are available to generate PROTACs. The human genome encodes more than 600 E3 ligases[6] and only a few of them (CRBN[1b], VHL[1a], cIAPs[7], and MDM2[8]) have been utilized by PROTACs to degrade POIs. This limits the ability to 
generate PROTACs for a POI that is not a suitable neo-substrate for those E3 ligases because different proteins may require different E3 ligases to effectively mediate their degradation. For example, endogenous $\mathrm{KRAS}^{\mathrm{G} 12 \mathrm{C}}$ can be degraded by VHL-recruiting PROTACs[9] rather than CRBN-recruiting PROTACs[10]. In addition, some E3 ligases are highly expressed in certain tumor cells[11], which may provide the opportunity to selectively degrade POIs in those tumor cells if ligands to recruit those E3 ligases can be found. Recent studies have also shown that cancer cells develop resistance to VHL-based bromodomain and extra-terminal domain (BET) PROTACs due to loss of CUL2 and to CRBN-based BET and CDK9 PROTACs because of CRBN loss[12]. Therefore, significant efforts have been devoted to finding new E3 ligase ligands, resulting in the discovery of ligands that can recruit AhR[13], DCAF11[14], DCAF15[15], DCAF16[16], KEAP1[17], RNF114[18], and RNF4[19] E3 ligases to degrade POIs. Identifying more E3 ligase ligands can further expand the toolbox, overcome the drug resistance, and potentially generate more potent and specific PROTACs.

Piperlongumine (PL, Figure 1A) is a natural product that exhibits potent antitumor activity[20], in part via induction of oxidative stress through its two Michael acceptors that can covalently react with GSTP1[21] and GSTO1[22]. Our previous studies also showed that PL can selectively kill senescent cells in part through induction of OXR1 degradation in a proteasome-dependent manner[23]. In addition, we found that PL can bind a number of intracellular proteins in senescent cells, including 8 different E3 ligases[23a], suggesting that PL could be used as a novel E3 ligase ligand to generate PROTACs. To test this hypothesis, we linked PL with SNS-032, a CDK9 inhibitor, and discovered that the PL-SNS-032 conjugates potently induced CDK9 degradation. Mechanistically, we demonstrated that the lead PL-SNS-032 conjugate, 955, degraded CDK9 in a ubiquitin-proteasome system (UPS) dependent manner. Using the TurboIDbait assay we identified KEAP1 as the E3 ligase recruited by 955 to degrade CDK9. Furthermore, a PL-based ALK PROTAC can potently degrade ALK-fusion protein in 
ALK positive non-small cell lung cancer (NSCLC) cells. Our results demonstrate that PL can be used as a new E3 ligase ligand to generate effective antitumor PROTACs.

\section{Results and Discussion}

\subsection{Validation of PL-binding E3 ligases}

Our previous study showed that PL can pull down 8 E3 ligases in senescent cells[23a]. To validate that PL covalently binds E3 ligases in cancer cells, we used a competitive activity-based protein profiling (ABPP) assay with the PL-Alkyne[23a] (Figure 1A) as a probe. Specifically, MOLT4 human T-cell acute lymphoblastic leukemia (T-ALL) cells were incubated with PL-Alkyne for $4 \mathrm{~h}$, then the cells were lysed and the cell lysates were used to perform a copper-catalysed azide-alkyne cycloaddition (CuAAC) reaction with Biotin-Azide. The PL-biotin-labelled proteins were pulled down by streptavidin beads and were eluted and detected by immunoblot or directly digested on the beads by trypsin for the identification of PL-binding proteins using liquid chromatography with tandem mass spectrometry (LC-MS/MS) (Figure 1B). To exclude proteins that bound non-specifically to PL-Alkyne, we also include a sample in which the cells were pre-treated with a high concentration of PL prior to the addition of the PL-Alkyne probe to compete for protein binding (Figure 1B). The western blot result showed that the PL-Alkyne probe can pull down many proteins, which can be effectively competed by the pre-treatment with PL (Figure 1C), suggesting that most of the pull-downed proteins by the PL-Alkyne probe are PL-binding proteins. The MS results showed that PL can recruit about 300 proteins (Figure 1D and Table S1), including GSTO1, a previously identified PL target[22]. We performed gene ontology (GO) and Kyoto encyclopedia of genes and genomes (KEGG) pathway enrichment analyses of those PL-binding proteins and found that many of the proteins involved in the UPS were highly enriched (Figure S1 and Table S1), including 9 E3 ligases (Figure 1D). These findings indicate that PL has the potential to be used for PROTAC design through recruiting E3 ligase(s). 


\subsection{Generation of PL-conjugated CDK9 PROTACs}

Based on the findings from the competitive ABPP assay, we hypothesized that PL can be used as a covalent E3 ligase ligand to generate PROTACs for degradation of POIs. CDK9 is a well-established cancer target and can be effectively degraded by a PROTAC (THAL-SNS-032) consisting of thalidomide, a linker, and the CDK9 selective inhibitor SNS-032[24]. Therefore, to test our hypothesis, we generated a series of PL-SNS-032 bifunctional molecules with linkers of different types and lengths (Figure S2A, B). PARP cleavage and cell viability assays were used to evaluate the potency of those compounds in induction of CDK9 degradation and apoptosis in MOLT4 cells (Figure S2B, C) and 955 (Figure 2A) was selected as a lead compound for further evaluation and characterization because of its high potency in these assays. 955 can potently degrade $\mathrm{CDK} 9$ with a $\mathrm{DC}_{50}$ of $9 \mathrm{nM}$ after $16 \mathrm{~h}$ treatment in MOLT4 cells (Figure S2B, C). Even with a short-term treatment $(6$ h), 955 can still potently degrade CDK9 while the warhead SNS-032 cannot (Figure 2B). In addition, 955 is more potent in inducing PARP cleavage than SNS-032 (Figure 2B). The time-course study showed that CDK9 can be completely degraded by $0.1 \mu \mathrm{M} 955$ within $8 \mathrm{~h}$ (Figure 2C) and the effect can last up to $18 \mathrm{~h}$ after the removal of 955 from the culture (Figure 2D). Similar results were also observed in $293 \mathrm{~T}$ cells (Figure S3A) and K562 cells (Figure S3B). To exclude the possibility that the degradation of CDK9 is caused by the combination effect of PL and SNS-032, we treated MOLT4 cells with either PL or SNS032 alone or their combination and found that none of these treatments degrades CDK9 (Figure 2E). However, pre-treatment of MOLT4 cells with PL or SNS-032 can block 955-induced CDK9 degradation (Figure 2F).

\subsection{Mechanism of action of 955 in degrading CDK9}

To confirm that 955 functions as a PROTAC to degrade CDK9, we performed a series of mechanistic studies using inhibitors to block different protein degradation pathways. First, we treated MOLT4 cells with the proteasome inhibitor MG132 or Bortezomib or 
with vehicle prior to 955 treatment. The results showed that the degradation of CDK9 can be blocked by the two different proteasome inhibitors (Figure 3A). In contrast, pretreatment with either of the two lysosome inhibitors, Baf-A1 and Chloroquine, or with the pan-caspase inhibitor QVD cannot block CDK9 degradation by 955 (Figure 3B, C). These results demonstrate that 955 induces CDK9 degradation through the proteasome but not lysosome and activated caspases. Furthermore, we used two E1 inhibitors, PYR41 and TAK-243, to verify that 955-induced CDK9 degradation is E1-dependent (Figure 3D). In our competitive ABPP assay, 9 PL-recruited E3 ligases were identified by the LC-MS/MS (Figure 1D), and thus we next investigated which type of E3 ligase(s) is involved in the degradation of CDK9 by 955 . MLN4924 is a NEDD8activating enzyme (NAE) inhibitor and can selectively inhibit cullin RING-related ubiquitin E3 ligase(s) (CRLs) through blocking the neddylation of cullin[25]. We found that MLN4924 pre-treatment blocks 955-induced CDK9 degradation (Figure 3E), suggesting that a CRL is likely to be recruited by 955 to degrade CDK9. Similar results were also observed in $293 \mathrm{~T}$ and K562 cells (Figure S4). Collectively, these findings suggest that 955 degrades CDK9 in a UPS-dependent manner probably via recruiting a CRL. Furthermore, we investigated whether 955 degrading CDK9 relies on PL to covalently recruit an E3 ligase via its two Michael acceptors by generating 336, in which the C2-3 and C7-8 double bonds of PL are reduced (Figure 3F). We found that 955, but not 336, can induce CDK9 degradation (Figure 3G), indicating that 955 degrades CDK9 via covalently recruiting a CRL through PL's Michael acceptors.

\subsection{Identification of 955-recruited E3 ligase(s) for CDK9 degradation}

PROTACs induce degradation of target proteins by hijacking an E3 ligase and it is difficult to directly identify the E3 ligase in the ternary complex recruited by the E3 ligase ligand in a PROTAC using the co-immunoprecipitation method because the formation of the complex is transient and very dynamic[26]. TurboID assay is a powerful proximity labeling method that can sensitively detect weak and transient protein interactions by biotinylating proteins that interact with a bait protein fused with 
an engineered biotin ligase[27]. Similar biotin-based proximity labeling assays, such as APEX2 assay[28] and AirID assay[29], have been used to study the degrader-induced or inhibitor-blocked E3:substrate interactions in cells. Therefore, we adapted TurboID technology to identify and characterize the specific E3 ligase(s) recruited by 955 to mediate CDK9 degradation by ectopically expressing V5-TurboID-CDK9 in 293T cells and comparing the biotinylated proteins with or without 955 treatment (Figure 4A). The results from this assay showed that $\mathbf{9 5 5}$ can induce the interaction of CDK9 with 6 different E3 ligases (Figure 4B, Table S2). To compare the PL-binding E3 ligases identified by the competitive ABPP assay (Figure 1D) with these identified by the TurboID-bait assay (Figure 4B), we found that KEAP1 is the only CRL identified by both methods. A previous study showed that KEAP1 can be recruited to degrade Tau protein by a KEAP1-based peptide PROTAC[30]. In addition, two recent studies discovered KEAP1 E3 ligase ligands that can be used to recruit KEAP1 to degrade BRD4 after linking to the BRD4 inhibitor JQ1[17]. To determine whether KEAP1 is the E3 ligase recruited by $\mathbf{9 5 5}$ to degrade CDK9, we first used immunoblot to confirm the results of the TurboID-bait assay. We found that significantly more KEAP1 can be pulled down after 955 treatment than without 955 treatment in the presence of biotin. By contrast, $\beta$-actin cannot be pulled down with or without 955 treatment under the same experimental conditions (Figure 4C). To further validate that KEAP1 is recruited by 955 to mediate CDK9 degradation, we treated MOLT4 cells with the known KEAP1 covalent inhibitors CDDO-ME, CDDO-IM, and dimethyl fumarate (DMF)[31], prior to the addition of $\mathbf{9 5 5}$. Such inhibitor treatment completely blocked 955-induced CDK9 degradation (Figure 4D, E). However, genetically proving that 955 recruits KEAP1 to degrade CDK9 has been difficult because KEAP1, as a sensor protein of oxidative and electrophilic stress[32], is essential for cell survival[33]. We have not been able to generate stable complete KEAP1 knockout cells using CRISPR technology. Furthermore, knockdown KEAP1 with shRNA cannot block CDK9 degradation by $\mathbf{9 5 5}$, because it has been reported that a low fractional occupancy of E3 ligase is sufficient to induce target degradation according to the mechanistic mathematical model of covalent E3 PROTACs developed by Chaudhry[34]. In addition, a recent study showed 
that even $10 \%$ of the normal level of DCAF16 was sufficient to mediate target degradation after it was recruited to induce protein degradation[16]. Therefore, we next investigated whether 955 recruits KEAP1 to degrade CDK9 by covalently binding to KEAP1. We performed a gel-based competitive ABPP assay, in which we competed serially diluted 955 against binding of a non-selective cysteine-reactive iodoacetamide (IA)-Alkyne probe to purified KEAP1 protein. The result from this assay confirms that 955 can compete with IA-Alkyne to covalently bind to KEAP1 in a concentrationdependent manner (Figure 4F). Forming a POI:PROTAC:E3 ternary complex is a necessary step for the induction of POI ubiquitination and degradation. Therefore, we fused CDK9 with HiBit-tag and KEAP1 with Halo-tag to perform nanoBRET assay to further test if 955 can induce the formation of the CDK9:955:KEAP1 ternary complex in live cells (Figure 4G). The results from this assay demonstrated that 955 can form a ternary complex with CDK9 and KEAP1 in a dose-dependent manner while $\mathbf{3 3 6}$ only shows very weak ternary complex formation, suggesting that the formation of this ternary complex is dependent on the covalent binding of 955 to KEAP1 via the PL Michael acceptor(s) (Figure 4G). In conclusion, 955 can covalently hijack KEAP1 to mediate CDK9 degradation.

\section{5 is a more potent anti-cancer agent than SNS-032}

To evaluate the anti-cancer efficacy of $\mathbf{9 5 5}$, a series of experiments were conducted to compare 955 with its warhead SNS-032. We first compared the global proteome changes induced by 955 and SNS-032 in MOLT4 cells. As expected, cells exhibit a significant reduction of CDK9 within $1 \mathrm{~h}$ after treatment with $0.1 \mu \mathrm{M} 955$ while treatment with $1 \mu \mathrm{M}$ SNS-032 for $6 \mathrm{~h}$ had no effect on CDK9 (Figure 5A and Table S3). Interestingly, 955, but not SNS-032, can also potently degrade CDK10 (Figure 5A, $\mathrm{B}$, and Table S3) in a proteasome- and CRL-dependent manner because pre-treatment with MG132 and MLN4924 can block the degradation (Figure 5C). This is a surprising finding because SNS-032 has a very low binding affinity to CDK10[24]. However, a similar phenomenon has been observed in a previously reported study[35], suggesting 
that sometimes a weak inhibitor/binder can be converted into a potent PROTAC degrader. Short time $(1 \mathrm{~h})$ treatment with $\mathbf{9 5 5}$ also reduces the expression of FYTTD1, which is likely a downstream target of CDK9 because its expression is reduced after SNS-032 treatment as well (Figure 5A). After a longer treatment (6 h), $0.1 \mu \mathrm{M} 955$ can be similarly effective in reducing the expression of a number of CDK9-regulated proteins as $1 \mu \mathrm{M}$ SNS-032, suggesting that 955 is more potent than its warhead SNS032 in suppressing the expression of CDK9-regulated proteins (Figure 5A and Figure S5). CDK9 plays a key role in regulating gene transcription by interacting with RNA polymerase II[36]. Therefore, we further performed RNA-seq to analyze the transcriptional changes in the same samples used for the proteome profiling. The results from this analysis confirmed that $\mathbf{9 5 5}$ is more potent than SNS-032 in down-regulation of CDK9-dependent transcription in MOLT4 cells after $6 \mathrm{~h}$ treatment (Figure 5D, E, Figure S6, and Table S4). c-Myc and MCl-1 are two well-known downstream targets of CDK9[36]. Quantitative real-time PCR (qPCR) and immunoblot revealed that 955 is about 10-fold more potent than SNS-032 in downregulation of c-Myc and $\mathrm{MCl}-1$ expression at the levels of both mRNA and protein (Figure 5F, G). We further compared the anti-proliferative effect of $\mathbf{9 5 5}$ with SNS-032 in prostate cancer cell lines since previous studies showed that targeting CDK9 is an effective way to inhibit prostate cancer[36]. Two AR-positive (LNCaP and 22RV1) and two AR-negative (PC3 and DU145) cell lines were studied. We found that 955 exhibits antitumor activities against all four cell lines with $\mathrm{EC}_{50}$ values in the single digital $\mathrm{nM}$ range, while the $\mathrm{EC}_{50}$ values of SNS-032 against these cells lines are over 100 nM (Figure 5H). Furthermore, immunoblot assays showed that $\mathbf{9 5 5}$ potently degraded CDK9 and downregulated cMyc and MCL-1 in LNCaP cells (Figure 5I). Collectively, these results indicate that $\mathbf{9 5 5}$ is more potent than SNS-032 against tumor cells.

\subsection{A PL-Ceritinib conjugate degrades ALK-fusion oncoprotein}

To further evaluate the potential of PL as a novel covalent E3 ligase ligand to generate PROTACs for the degradation of oncoproteins, we synthesized 819 by linking PL to 
Ceritinib for the degradation of EML4-ALK, an oncogenic fusion protein for NSCLC (Figure 6A) because patients with EML4-ALK-positive metastatic NSCLC frequently develop resistance to the anaplastic lymphoma kinase (ALK) inhibitor Ceritinib[37]. In addition, Ceritinib has been used for generating a CRBN-ALK PROTAC[38]. We found that $\mathbf{8 1 9}$ degrades EML4-ALK in a concentration-dependent manner in NCIH2228 NSCLC cells (Figure 6B). The degradation of EML4-ALK can be blocked by the pre-treatment of the cells with MG132, MLN4924, and DMF (Figure 6C, D), suggesting that $\mathbf{8 1 9}$ may also recruit KEAP1 to mediate the degradation of EML4-ALK in NCI-H2228 cells. With further optimization of the linker, we expect that PLCeritinib conjugates can more potently degrade EML4-ALK in NSCLC cells.

\section{Conclusion}

Despite the human proteome encoding more than 600 E3 ligases[6], only a few E3 ligases have been used for PROTAC design due to the limited availability of E3 ligase ligands. Therefore, finding new E3 ligase ligands to expand the toolbox of PROTAC technology is critical for further development of this field. Significant progress has been made in this regard in the last several years, resulting in the discovery of a number of new E3 ligase ligands[4], including some covalent ones that can recruit DCAF11[14], DCAF16[16], KEAP1[17b], RNF114[18], and RNF4[19] E3 ligases for PROTAC designing and protein degradation. Based on mathematical modeling[34] and previous studies[16-17, 18a, 39], covalent E3 ligase ligand-based PROTACs may outperform non-covalent E3 ligase ligand-based PROTACs due to better kinetics of ternary complex formation, and minimal perturbation of its endogenous substrates with low fractional occupancy of the recruited E3 ligase. In addition, accumulating evidence shows that a covalent E3 ligase ligand in a PROTAC provides additional selectivity for a given POI[16, 18a, 19]. In this study, we design and synthesize a series of PL-SNS032 conjugates and find that the lead compound of PL-SNS-032 conjugates, 955, induces potent proteasomal degradation of CDK9. To find the E3 ligase(s) engaged by 955, we use the TurboID-bait assay to identify the proteins that transiently interact with 
CDK9 induced by $\mathbf{9 5 5}$, finding that KEAP1 is the E3 ligase recruited by 955 through covalent binding of PL. We further demonstrate that EML4-ALK protein can also be successfully degraded by the PL-Ceritinib conjugate 819, which provides additional evidence to demonstrate that PL can be used as a new KEAP1 ligand to induce the degradation of different POIs. Compared with the other two commonly used E3 ligases (VHL and CRBN), KEAP1 is highly expressed in many tumor cells such as lung, kidney, breast, prostate, and brain cancer cells[17a]. Therefore, PL-based PROTACs may achieve better degradation efficacy in those cancer cells. Additionally, with a relatively smaller molecular size (MW=317.3 Da), PL-based PROTACs might possess more favorable physicochemical properties for drug development. However, it requires further investigation to characterize the binding mode of PL with KEAP1, which can guide optimization of PL as a useful E3 ligase ligand for the design of PROTACs. For example, we need to find which of the two Michael acceptors in PL plays the major role to recruit KEAP1. In addition, it will be important to determine whether the reactivity of the Michael acceptors can be modulated to increase its specificity in binding KEAP1 while reducing its non-specifical binding to other proteins. In conclusion, our study along with several recently published reports[18] demonstrates that natural products are an important source for the discovery of new E3 ligase ligands. In addition, our studies also show that the TurboID-bait assay or other biotin-based proximity labeling assays[28-29] can be a powerful tool to identify E3 ligase(s) recruited by a new $\mathrm{E} 3$ ligase ligand.

\section{Experimental methods}

Materials and Methods, Synthetic Chemistry of $\mathbf{9 5 5}, \mathbf{3 3 6}, \mathbf{8 1 9}$, and all other degraders and intermediates are detailed in the Supporting Information.

\section{Acknowledgements}

This study was supported in part by US National Institutes of Health (NIH) grants R01 CA242003 (D.Z. and G.Z.), R01 CA241191 (G.Z., M.K., and D.Z.), R01 AG063801 
(D.Z. and G.Z.) and R56AG065635 (G.Z.). A Leukemia and Lymphoma Society Specialzied Center of Resaerch (J.D.L.) and LLS Special Fellow Award (A.S). We thank the Mass Spectrometry Research and Education Center and the funding source: NIH S10 OD021758-01A1. The authors would like to thank Ms. Janet Wiegand and Alexandra M. Fahnlander for editing the manuscript.

\section{Conflict of interest}

J.P., Y.X., X.L., W.H., Y.Y., G.Z., D.Z., and D.L. are co-inventors of a pending patent application for the discovery of PL as an E3 ligase ligand. G.Z. and D.Z. are co-founders and have equity of Dialectic Therapeutics that develops BCL-xL/2 PROTACs for cancer treatment. J.D.L. is a scientific advisor to Dialectic Therapeutics. All other authors declare no conflict of interest.

\section{Author information}

Corresponding Authors:

Guangrong Zheng - Department of Medicinal Chemistry, College of Pharmacy, University of Florida, 1333 Center Drive, Gainesville, FL, 32610, United States; Email: zhengg@ cop.ufl.edu.

Daohong Zhou - Department of Pharmacodynamics, College of Pharmacy, University of Florida, 1333 Center Drive, Gainesville, FL, 32610, United States; E-mail: zhoudaohong@cop.ufl.edu

Dongwen Lv - Department of Pharmacodynamics, College of Pharmacy, University of Florida, 1333 Center Drive, Gainesville, FL, 32610, United States; E-mail: lyudongwen@cop.ufl.edu

\section{References}

[1] a) D. P. Bondeson, A. Mares, I. E. Smith, E. Ko, S. Campos, A. H. Miah, K. E. Mulholland, N. Routly, D. L. Buckley, J. L. Gustafson, Nature chemical biology 2015, 11 (8), 611; b) G. E. Winter, D. L. Buckley, J. Paulk, J. M. Roberts, A. Souza, S. DhePaganon, J. E. Bradner, Science 2015, 348 (6241), 1376.

[2] L. Bai, H. Zhou, R. Xu, Y. Zhao, K. Chinnaswamy, D. McEachern, J. Chen, C.-Y. Yang, 
Z. Liu, M. Wang, Cancer cell 2019, 36 (5), 498.

[3] a) S. Khan, X. Zhang, D. Lv, Q. Zhang, Y. He, P. Zhang, X. Liu, D. Thummuri, Y. Yuan, J. S. Wiegand, Nature medicine 2019, 25 (12), 1938; b) D. Lv, P. Pal, X. Liu, Y. Jia, D. Thummuri, P. Zhang, W. Hu, J. Pei, Q. Zhang, S. Zhou, Nature Communications 2021, 12 (1), 1; c) M. S. Gadd, A. Testa, X. Lucas, K.-H. Chan, W. Chen, D. J. Lamont, M. Zengerle, A. Ciulli, Nature chemical biology 2017, 13 (5), 514.

[4] A. Mullard, Nature reviews. Drug Discovery 2021.

[5] H. Gao, X. Sun, Y. Rao, ACS medicinal chemistry letters 2020, 11 (3), 237.

[6] W. Li, M. H. Bengtson, A. Ulbrich, A. Matsuda, V. A. Reddy, A. Orth, S. K. Chanda, S. Batalov, C. A. Joazeiro, PloS one 2008, 3 (1), e1487.

[7] M. Naito, N. Ohoka, N. Shibata, Drug Discovery Today: Technologies 2019, 31, 35.

[8] J. Hines, S. Lartigue, H. Dong, Y. Qian, C. M. Crews, Cancer research 2019, 79 (1), 251.

[9] M. J. Bond, L. Chu, D. A. Nalawansha, K. Li, C. M. Crews, ACS central science 2020, $6(8), 1367$.

[10] M. Zeng, Y. Xiong, N. Safaee, R. P. Nowak, K. A. Donovan, C. J. Yuan, B. Nabet, T. W. Gero, F. Feru, L. Li, Cell Chemical Biology 2020, 27 (1), 19.

[11] Y. He, S. Khan, Z. Huo, D. Lv, X. Zhang, X. Liu, Y. Yuan, R. Hromas, M. Xu, G. Zheng, Journal of hematology \& oncology 2020, 13 (1), 1.

[12] a) R. Shirasaki, G. M. Matthews, S. Gandolfi, R. de Matos Simoes, D. L. Buckley, J. R. Vora, Q. L. Sievers, J. B. Brüggenthies, O. Dashevsky, H. Poarch, Cell Reports 2021, 34 (1), 108532; b) L. Zhang, B. Riley-Gillis, P. Vijay, Y. Shen, Molecular cancer therapeutics 2019, 18 (7), 1302.

[13]N. Ohoka, G. Tsuji, T. Shoda, T. Fujisato, M. Kurihara, Y. Demizu, M. Naito, ACS chemical biology 2019, 14 (12), 2822.

[14]X. Zhang, L. M. Luukkonen, C. L. Eissler, V. M. Crowley, Y. Yamashita, M. A. Schafroth, S. Kikuchi, D. S. Weinstein, K. T. Symons, B. E. Nordin, Journal of the American Chemical Society 2021, 143 (13), 5141.

[15] V. Zoppi, S. J. Hughes, C. Maniaci, A. Testa, T. Gmaschitz, C. Wieshofer, M. Koegl, K. M. Riching, D. L. Daniels, A. Spallarossa, Journal of medicinal chemistry 2018, 62 (2), 699.

[16]X. Zhang, V. M. Crowley, T. G. Wucherpfennig, M. M. Dix, B. F. Cravatt, Nature chemical biology 2019, 15 (7), 737.

[17] a) J. Wei, F. Meng, K.-S. Park, H. Yim, J. Velez, P. Kumar, L. Wang, L. Xie, H. Chen, Y. Shen, Journal of the American Chemical Society 2021, 143 (37), 15073; b) B. Tong, M. Luo, Y. Xie, J. N. Spradlin, J. A. Tallarico, J. M. McKenna, M. Schirle, T. J. Maimone, D. K. Nomura, Scientific reports 2020, 10 (1), 1.

[18] a) J. N. Spradlin, X. Hu, C. C. Ward, S. M. Brittain, M. D. Jones, L. Ou, M. To, A. Proudfoot, E. Ornelas, M. Woldegiorgis, J. A. Olzmann, D. E. Bussiere, J. R. Thomas, J. A. Tallarico, J. M. McKenna, M. Schirle, T. J. Maimone, D. K. Nomura, Nat Chem Biol 2019, 15 (7), 747, https://doi.org/10.1038/s41589-019-0304-8; b) M. Luo, J. N. Spradlin, L. Boike, B. Tong, S. M. Brittain, J. M. McKenna, J. A. Tallarico, M. Schirle, T. J. Maimone, D. K. Nomura, Cell Chemical Biology 2021, 28 (4), 559.

[19] C. C. Ward, J. I. Kleinman, S. M. Brittain, P. S. Lee, C. Y. S. Chung, K. Kim, Y. Petri, 
J. R. Thomas, J. A. Tallarico, J. M. McKenna, M. Schirle, D. K. Nomura, ACS Chem Biol 2019, 14 (11), 2430, https://doi.org/10.1021/acschembio.8b01083.

[20] J.-L. Roh, E. H. Kim, J. Y. Park, J. W. Kim, M. Kwon, B.-H. Lee, Oncotarget 2014, 5 (19), 9227.

[21] W. Harshbarger, S. Gondi, S. B. Ficarro, J. Hunter, D. Udayakumar, D. Gurbani, W. D. Singer, Y. Liu, L. Li, J. A. Marto, Journal of Biological Chemistry 2017, 292 (1), 112.

[22] L. Li, Y. Zhao, R. Cao, L. Li, G. Cai, J. Li, X. Qi, S. Chen, Z. Zhang, Chemical Communications 2019, 55 (30), 4407.

[23] a) X. Zhang, S. Zhang, X. Liu, Y. Wang, J. Chang, X. Zhang, S. G. Mackintosh, A. J. Tackett, Y. He, D. Lv, Aging cell 2018, 17 (4), e12780; b) Y. Wang, J. Chang, X. Liu, X. Zhang, S. Zhang, X. Zhang, D. Zhou, G. Zheng, Aging (Albany NY) 2016, 8 (11), 2915.

[24] C. M. Olson, B. Jiang, M. A. Erb, Y. Liang, Z. M. Doctor, Z. Zhang, T. Zhang, N. Kwiatkowski, M. Boukhali, J. L. Green, Nature chemical biology 2018, 14 (2), 163.

[25] a) T. Kawakami, T. Chiba, T. Suzuki, K. Iwai, K. Yamanaka, N. Minato, H. Suzuki, N. Shimbara, Y. Hidaka, F. Osaka, The EMBO journal 2001, 20 (15), 4003; b) E. Sakata, Y. Yamaguchi, Y. Miyauchi, K. Iwai, T. Chiba, Y. Saeki, N. Matsuda, K. Tanaka, K. Kato, Nature structural \& molecular biology 2007, 14 (2), 167.

[26]X. Liu, X. Zhang, D. Lv, Y. Yuan, G. Zheng, D. Zhou, Future Medicinal Chemistry 2020, 12 (12), 1155.

[27] T. C. Branon, J. A. Bosch, A. D. Sanchez, N. D. Udeshi, T. Svinkina, S. A. Carr, J. L. Feldman, N. Perrimon, A. Y. Ting, Nature biotechnology 2018, 36 (9), 880.

[28] C. Mayor-Ruiz, M. G. Jaeger, S. Bauer, M. Brand, C. Sin, A. Hanzl, A. C. Mueller, J. Menche, G. E. Winter, Molecular cell 2019, 75 (4), 849.

[29] K. Kido, S. Yamanaka, S. Nakano, K. Motani, S. Shinohara, A. Nozawa, H. Kosako, S. Ito, T. Sawasaki, Elife 2020, 9, e54983.

[30] M. Lu, T. Liu, Q. Jiao, J. Ji, M. Tao, Y. Liu, Q. You, Z. Jiang, European journal of medicinal chemistry 2018, 146, 251.

[31] T. Suzuki, M. Yamamoto, Journal of Biological Chemistry 2017, 292 (41), 16817.

[32]D. D. Zhang, S.-C. Lo, J. V. Cross, D. J. Templeton, M. Hannink, Molecular and cellular biology 2004, 24 (24), 10941.

[33] N. Wakabayashi, K. Itoh, J. Wakabayashi, H. Motohashi, S. Noda, S. Takahashi, S. Imakado, T. Kotsuji, F. Otsuka, D. R. Roop, Nature genetics 2003, 35 (3), 238.

[34]C. Chaudhry, 2021.

[35]D. P. Bondeson, B. E. Smith, G. M. Burslem, A. D. Buhimschi, J. Hines, S. JaimeFigueroa, J. Wang, B. D. Hamman, A. Ishchenko, C. M. Crews, Cell chemical biology 2018, 25 (1), 78.

[36] M. H. Rahaman, M. Kumarasiri, L. B. Mekonnen, M. Yu, S. Diab, H. Albrecht, R. W. Milne, S. Wang, Endocrine-related cancer 2016, 23 (12), T211.

[37] Y. Pan, C. Deng, Z. Qiu, C. Cao, F. Wu, Frontiers in Oncology 2021, 3945.

[38] C. Zhang, X.-R. Han, X. Yang, B. Jiang, J. Liu, Y. Xiong, J. Jin, European journal of medicinal chemistry 2018, 151, 304.

[39] a) R. Gabizon, N. London, Current Opinion in Chemical Biology 2021, 62, 24; b) H. Kiely-Collins, G. E. Winter, G. J. Bernardes, Cell Chemical Biology 2021. 


\section{Figures}

A<smiles>C#CCOc1c(OC)cc(/C=C/C(=O)N2CC=CC(=O)C2=O)cc1OCCCC</smiles>

B

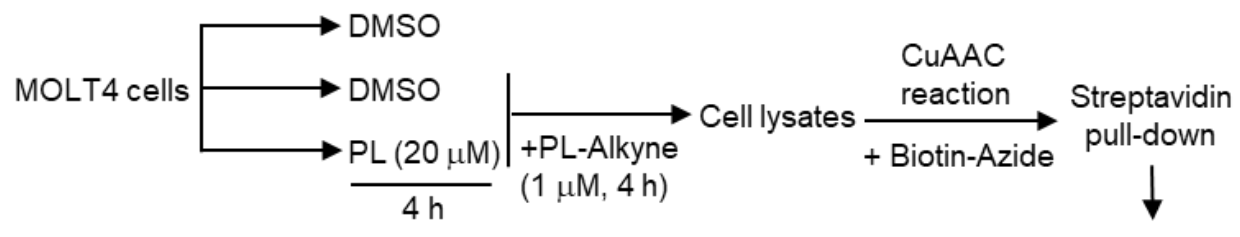

C

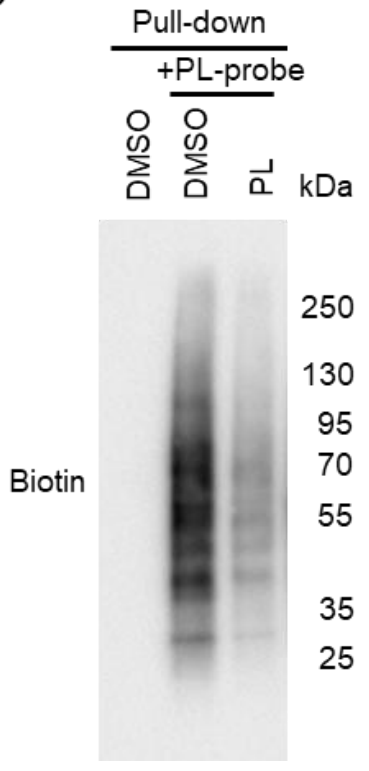

WB and quantitative proteomics

Figure 1. Validation of PL-binding E3 ligases. (A) The structures of PL and PLAlkyne probe; (B) The workflow of the competitive ABPP assay; (C) Western blot to detect biotin-labelled proteins in the streptavidin pull-downed samples; and (D) The PL-binding proteins identified by mass spectrometry. E3 ligases are highlighted in red and GSTO1 is shown in green. MOLT4 is a human T-cell acute lymphoblastic leukemia (T-ALL) cell line. 
A

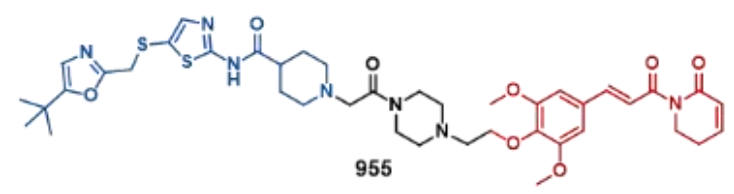

C

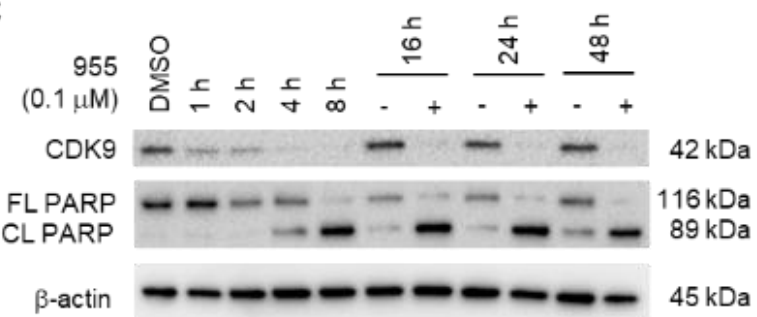

B

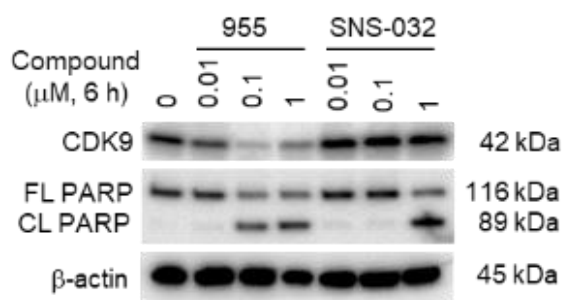

E

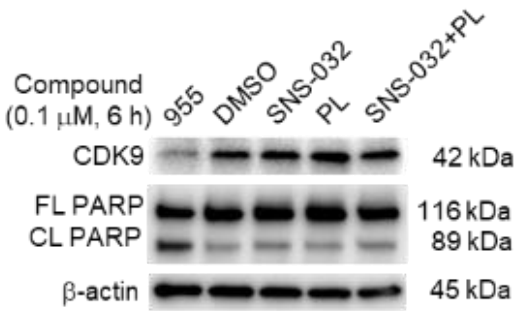

D

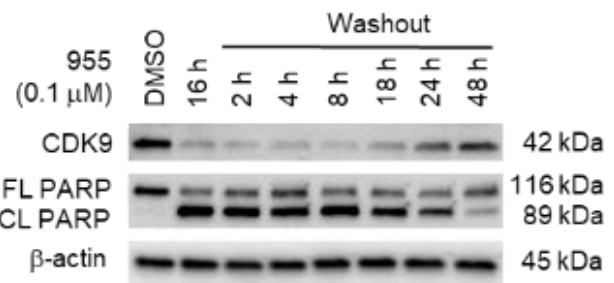

$\mathrm{F}$

$955(0.1 \mu \mathrm{M}, 6 \mathrm{~h}) \cdot+\cdot+\cdot+$

SNS-032 $(5 \mu \mathrm{M}, 2 \mathrm{~h}) \cdot-+\quad+\cdot$

$\mathrm{PL}(10 \mu \mathrm{M}, 2$ h) -

CDK9

$42 \mathrm{kDa}$

$\beta$-actin

$45 \mathrm{kDa}$

Figure 2. The lead PL-SNS-032 conjugate 955 potently induces CDK9 degradation

and apoptosis. (A) The structure of 955; (B) 955 but not SNS-032 induces the degradation of CDK9 and is more potent than SNS-032 in induction of apoptosis; (C) Time course of 955-induced CDK9 degradation; (D) CDK9 degradation lasts up to 18 $\mathrm{h}$ after washout of 955; (E) The combination of SNS-032 and PL cannot induce CDK9 degradation; and (F) Pre-treatment with SNS-032 or PL blocks the CDK9 degradation induced by 955. Representative immunoblots are shown and $\beta$-actin was used as a loading control in all immunoblot analyses. FL PARP (full-length PARP) and CL PARP (cleaved PARP) are used as the indicators of apoptosis. All the experiments were done in MOLT4 cells. 
A

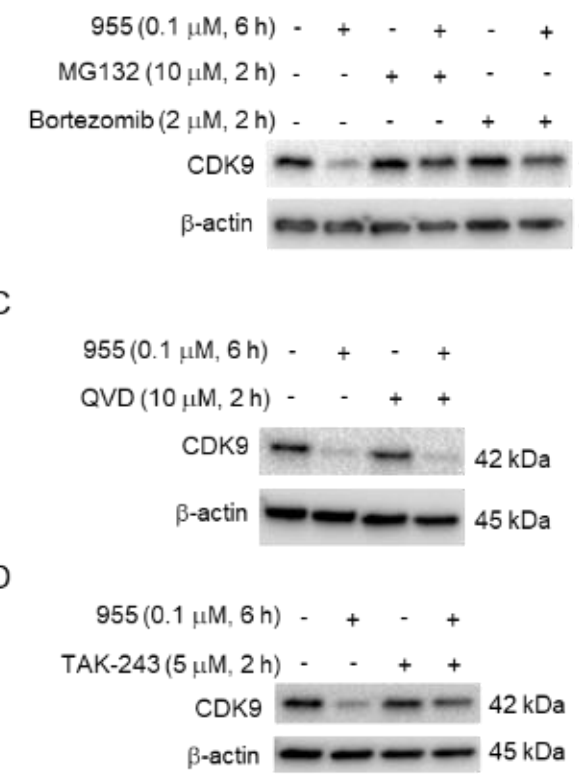

$\mathrm{F}$

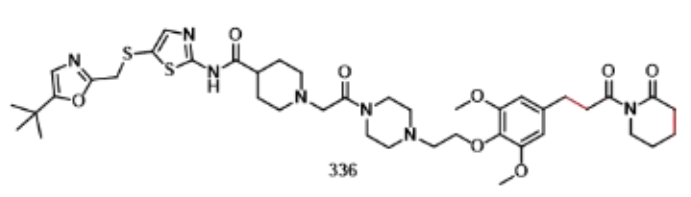

B

E
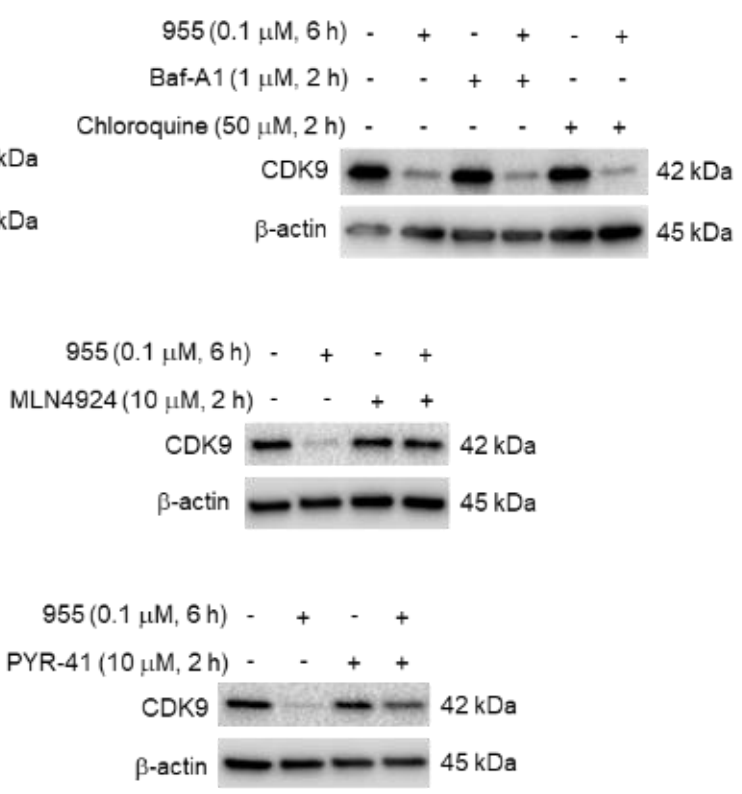

G

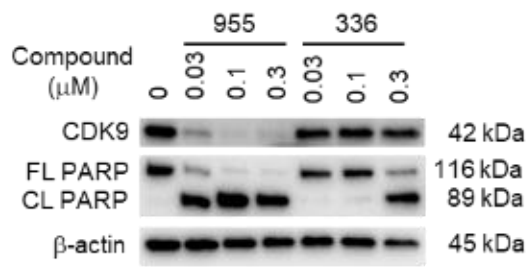

Figure 3. Mechanism of action of 955 in degrading CDK9. (A) Pre-treatment with the proteasome inhibitor MG132 or Bortezomib blocks CDK9 degradation by 955 ; (B) Pre-treatment with the lysosome inhibitor Baf-A1 or Chloroquine cannot block CDK9 degradation by 955; (C) Pre-treatment with pan-caspase inhibitor QVD cannot block CDK9 degradation by 955; (D) Pre-treatment with E1 inhibitor PYR-41 or TAK-243 blocks CDK9 degradation by 955; (E) Pre-treatment with neddylation inhibitor MLN4924 blocks CDK9 degradation by $\mathbf{9 5 5}$. (F) The structure of $\mathbf{3 3 6}$ to illustrate the reduction of the C2-3 and C7-8 double bonds of PL in comparison with 955; (G) 955, but not 336, can degrade CDK9. Representative immunoblots are shown and $\beta$-actin was used as a loading control in all immunoblot analyses. All the experiments were done in MOLT4 cells. 
A

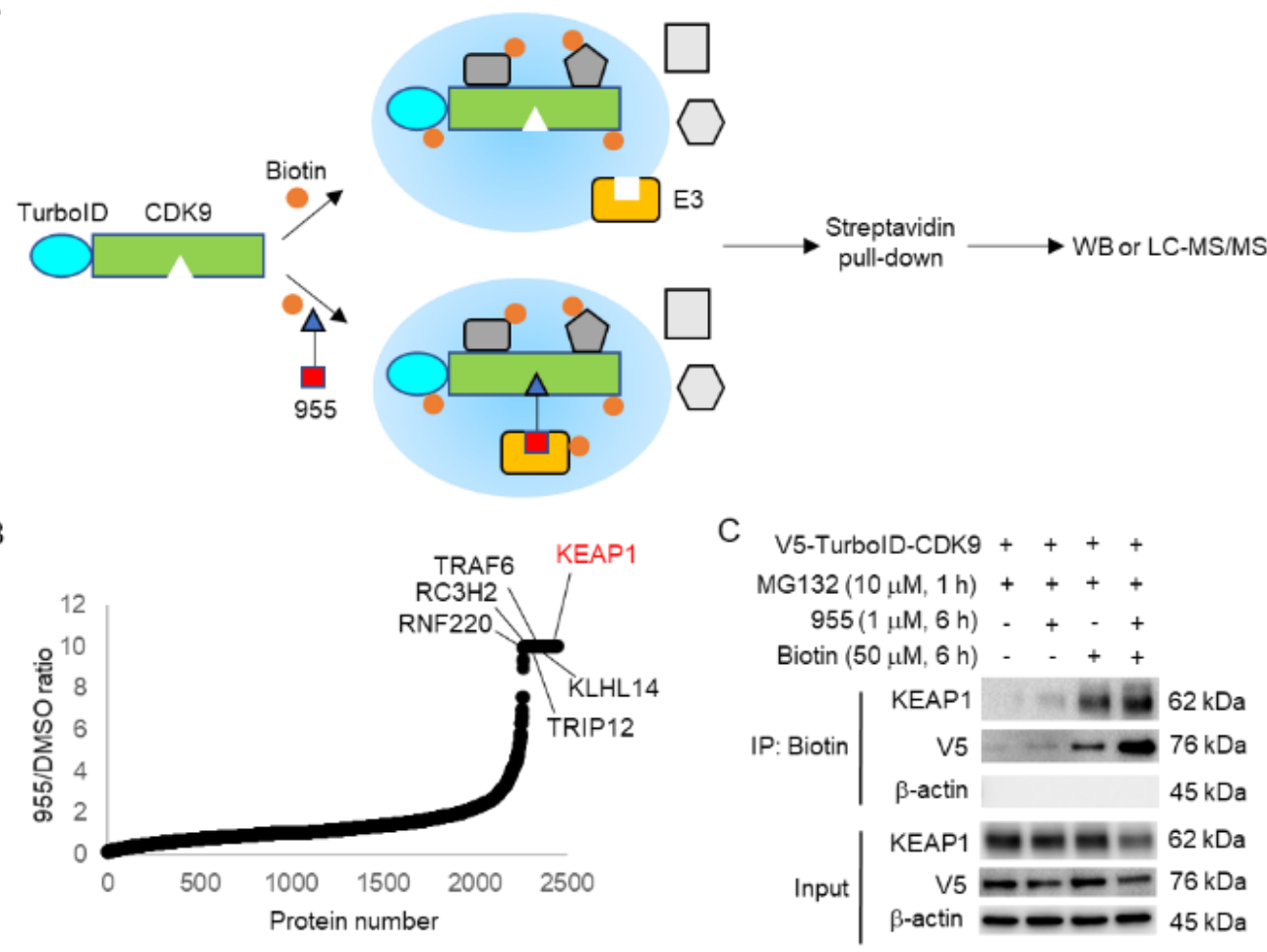

D

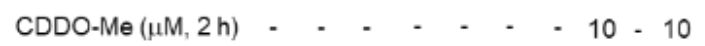

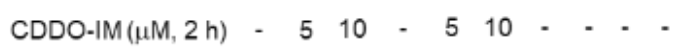

$955(0.1 \mu \mathrm{M}, 4 \mathrm{~h})-\ldots+++\cdots+$

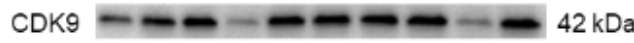

$\mathrm{E}$

$\operatorname{DMF}(2 \mathrm{~h}, \mu \mathrm{MM})-\cdots 501050$

$955(0.1 \mu \mathrm{M}, 6 \mathrm{~h}) \cdots+\cdots++$

CDK9 - $-\ldots-\cdots+42 \mathrm{kDa}$

$\beta$-actin

$\beta$-actin

$45 \mathrm{kDa}$

$\mathrm{F}$
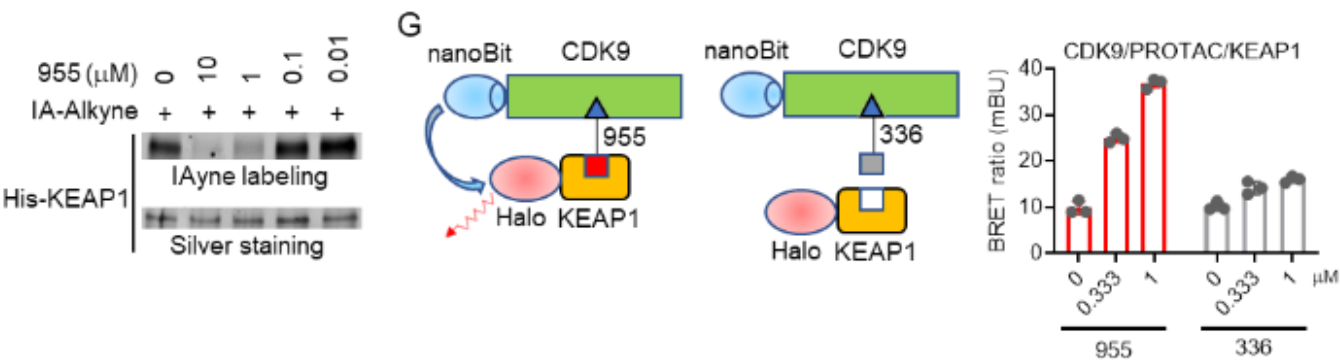

Figure 4. Identification of CDK9 degrading E3 ligase(s) recruited by 955 . (A)

Schematic illustration of the TurboID-bait assay to identify CDK9 degrading E3 ligase(s) recruited by 955; (B) The biotinylated proteins identified by MS in 293T cells. E3 ligases are labelled with KEAP1 highlighted in red; (C) Western blot analysis to validate KEAP1 recruitment by 955; (D) KEAP1 inhibition with CDDO-ME and CDDO-IM blocks 955-induced CDK9 degradation in MOLT4 cells; (E) Inhibition of KEAP1 with DMF blocks 955-induced CDK9 degradation in MOLT4 cells. 
Representative immunoblots are shown and $\beta$-actin was used as a loading control in all immunoblot analyses; (F) Gel-based ABPP assay demonstrates that 955 competes IAAlkyne binding to KEAP1 in a dose-dependent manner. (G) nanoBRET assay demonstrates that $\mathbf{9 5 5}$, but not $\mathbf{3 3 6}$, can induce the formation of the intracellular ternary complexes in live $293 \mathrm{~T}$ cells. Data are expressed as mean \pm s.e.m. of three biological replicates. 


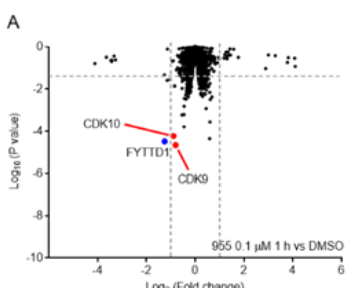

D

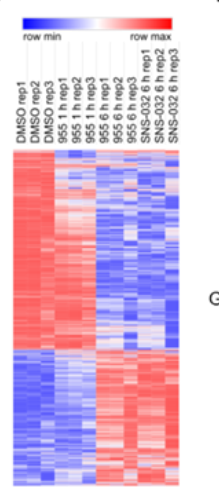

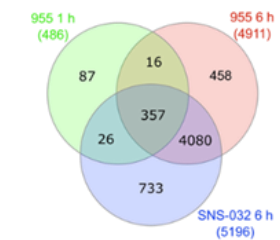

G

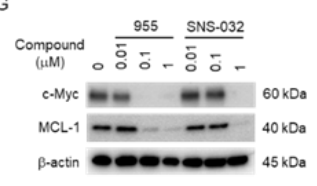

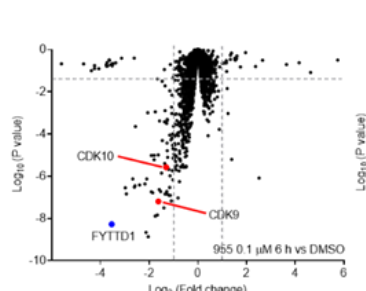

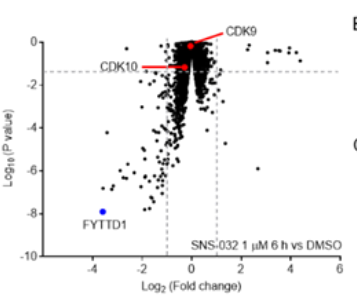

B
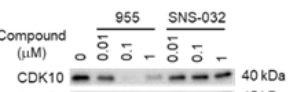

c

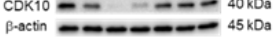

$955(0.1 \mu \mathrm{M}, 6 \mathrm{~h}) \ldots+\ldots+$

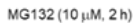

MLN4924 (10 $\mu \mathrm{M}, 2 \mathrm{~h})$

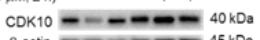

H
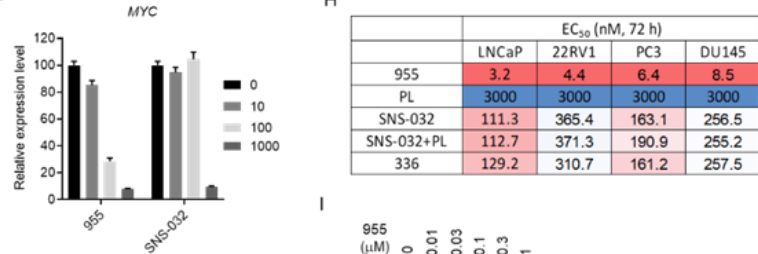

MCL

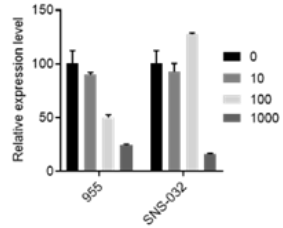

Figure 5.955 is a more potent antitumor agent than SNS-032. (A) Proteome changes induced by 955 or SNS-032 in MOLT4 cells; (B) 955, but not SNS-032, degrades CDK10 in MOLT4 cells. (C) Pre-treatment with MG132 and MLN4924 blocks CDK10 degradation induced by 955; (D) Heatmap and (E) Venn diagram to show the differentially expressed genes in MOLT4 cells after 955 and SNS-032 treatments; (F) and $(\mathrm{G})$ to demonstrate that the effects of 955 and SNS-032 on the expression of the two CDK9 downstream targets MYC and MCL1 in MOLT4 cells at the levels of transcription and translation determined by qPCR and immunoblot, respectively. The qPCR data are expressed as mean \pm s.e.m. of three biological replicates. (H) 955 exhibits higher potencies against multiple prostate cancer cells compared with PL, SNS-032, SNS-032 plus PL, and 336. The data are presented as mean from three independent experiments; (I) 955 induces CDK9 degradation and downregulates the expression of c-Myc and MCL-1 in LNCaP prostate cancer cells. Representative immunoblots are shown and $\beta$-actin was used as a loading control in all immunoblot analyses. 
A

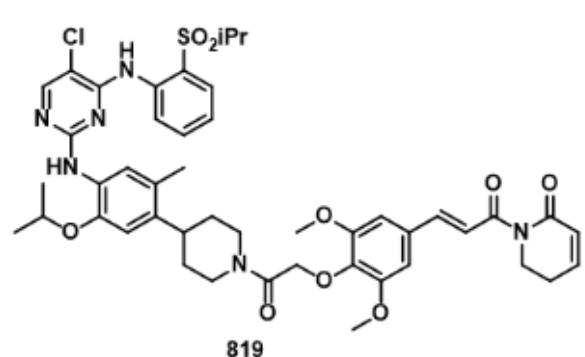

C

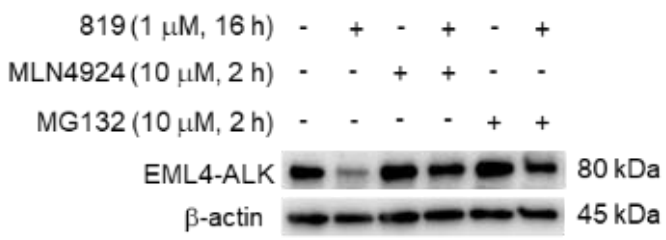

B

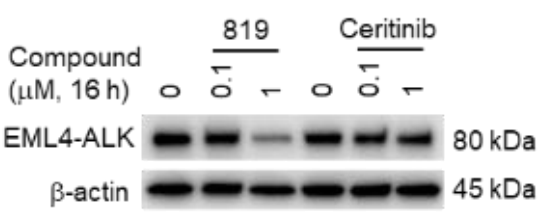

D

$\operatorname{DMF}(2 \mathrm{~h}, \mu \mathrm{M})$ - - 10501050 $819(0.3 \mu \mathrm{M}, 16 \mathrm{~h})$ -

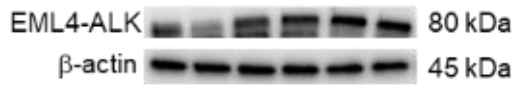

Figure 6. PL-Ceritinib conjugate degrades the EML4-ALK-fusion protein. (A) The structure of PL-Ceritinib conjugate 819; (B) 819 but not Ceritinib degrades EML4ALK in NCI-H2228 cells; (C) Pre-treatment with the neddylation inhibitor MLN4924 or proteasome inhibitor MG132 blocks EML4-ALK degradation induced by 819; and (D) Pre-treatment with the KEAP1 inhibitor DMF blocks the 819-induced EML4-ALK degradation. Representative immunoblots are shown and $\beta$-actin was used as a loading control in all immunoblot analyses. 